\title{
Delineating chiral separation effect in two-color dense QCD
}

\author{
Daiki Suenaga $\odot^{1, *}$ and Toru Kojo ${ }^{2, \dagger}$ \\ ${ }^{1}$ Research Center for Nuclear Physics, Osaka University, Ibaraki 567-0048, Japan \\ ${ }^{2}$ Key Laboratory of Quark and Lepton Physics (MOE) and Institute of Particle Physics, \\ Central China Normal University, Wuhan 430079, China
}

(Received 1 June 2021; accepted 6 August 2021; published 31 August 2021)

\begin{abstract}
We study the chiral separation effect (CSE) in two-color and two-flavor QCD to delineate quasiparticle pictures in dense matter from low to high temperatures. Both massless and massive quarks are discussed. We particularly focus on the high density domain where diquarks form a color-singlet condensate with the electric charge $1 / 3$. The condensate breaks the baryon number and $U(1)_{A}$ axial symmetry, and induces the electromagnetic Meissner effects. Within a quark quasiparticle picture, we compute the chiral separation conductivity at one-loop. We have checked that Nambu-Goldstone modes, which should appear in the improved vertices as required by the Ward-Takahashi identities, do not contribute to the chiral-separation conductivity due to their longitudinal natures. In the static limit, the destructive interferences in the particlehole channel, as in the usual Meissner effects, suppress the conductivity (in the chiral limit, to $1 / 3$ of that of the normal phase). This locally breaks the universality of the CSE coefficients, provided quasiparticle pictures are valid in the bulk matter.
\end{abstract}

DOI: $10.1103 /$ PhysRevD.104.034038

\section{INTRODUCTION}

The quantum anomaly [1-3] and topological properties of matter have been attracting much attention in a wide area of physics, due to their universal natures valid for abruptly different energy scales [4-10]. The nonrenormalization theorem for the anomaly relation [11-13], as well as the robustness of topology under local perturbations, are the key concepts for such universality.

The chiral conductivities are constrained by such universal relations, especially in the chiral limit (see e.g., a review [14]). One can also use the relations to constrain or enrich our pictures on the nonperturbative dynamics [15-22] which have important implications to the physics of neutron stars (for a review, see e.g., [23]). With this perspective in mind, in this paper we study the conductivity for the chiral separation effect (CSE) [24] and chiral separation (CS) conductivity, in two-color $\mathrm{QCD}$ with two-flavor $\left(\mathrm{QC}_{2} \mathrm{D}\right)$ at high baryon density. The relation is

$$
J_{i}^{5}=\bar{q} \gamma_{i} \gamma_{5} q=\frac{C}{2 \pi^{2}} \mu B_{i},
$$

\footnotetext{
suenaga@rcnp.osaka-u.ac.jp

torujj@mail.ccnu.edu.cn
}

Published by the American Physical Society under the terms of the Creative Commons Attribution 4.0 International license. Further distribution of this work must maintain attribution to the author(s) and the published article's title, journal citation, and DOI. Funded by SCOAP ${ }^{3}$. with $q$ quark fields, $\mu$ quark chemical potential, and $C$ being some constant related to the anomaly. The dense $\mathrm{QC}_{2} \mathrm{D}$ matter $[25,26]$ can be studied in lattice Monte-Carlo simulations [27-30] and hence can be used as laboratories to test the concepts and methodologies of dense QCD calculations [31-33]. The results may be extended to our main target- the three-color dense QCD.

The CSE in $\mathrm{QC}_{2} \mathrm{D}$ was studied for hadronic and quarkgluon-plasma phases [34], and was found to agree with theoretical predictions including explicit chiral symmetry breaking. Meanwhile, for cold dense matter the CSE has not been studied yet. At $\mu \geq m_{\pi} / 2$ ( $m_{\pi}$ is the pion mass), the dense matter has condensations of diquarks, $\sim u d$, which are color-singlet, $J^{P}=0^{+}$, and have electric charges of $1 / 3$. We call this phase the 'diquark condensed phase'. A number of new ingredients appear here, modifying qualitative pictures conventionally used to explain the CSE [24]; (i) A quark acquires the mass gap around the Fermi surface and arguments based on the lowest Landau level no longer directly apply, (ii) Diquarks are charged and should induce the electromagnetic Meissner effects, expelling magnetic fields from the bulk, and (iii) The spontaneous symmetry breaking demands the improved vertices to fulfill the WardTakahashi identity (WTI), and the vertices contain the poles of the Nambu-Goldstone (NG) modes [35], both in the axial vector and the vector vertices.

We address these qualitative issues within one-loop calculations in the linear response regime, as done for the chiral magnetic conductivity [36]. We assume the quasiparticle pictures for quarks with the gap $\Delta$, in a similar 
manner as in our previous calculations [31-33]. The CS conductivity in the static and dynamic limits will be analyzed for various temperatures. We find that the dependence on temperatures and the orders of static and dynamic limits can be understood through the particle-hole dynamics; in the chiral limit and at $T=0$, the static CS conductivity in the diquark condensed (normal) phase acquires $0(2 / 3)$ of the anomaly coefficient from the particle-hole contributions and $1 / 3(1 / 3)$ from particle-antiparticle contributions. Thus the static CS conductivity in the diquark condensed phases provides only $1 / 3$ of that expected from the universal relation. ${ }^{1}$

Inclusion of the NG modes through the improved vertices does not help as they are longitudinal modes and hence decouple. This suggests that, if the coefficient of the CS conductivity is indeed constrained by the anomaly, we should go beyond our quasiparticle descriptions or the linear response regime, e.g., by manifestly treating vortices with fermion zero modes or zero modes on the boundaries of the diquark condensed phase.

This paper is structured as follows. In Sec. II we set up our frameworks and introduce quasiparticle propagators in the diquark condensed phase. In Sec. III we summarize the expressions for the CS conductivity within the linear response regime. In Sec. IV we present our numerical results for various chemical potentials, temperatures, quark masses, and diquark gaps. In Sec. $\mathrm{V}$ we address the contributions not included in our quasiparticle calculations in the bulk. This paper is closed with summary in Sec. VI.

\section{MODEL}

Here we introduce an effective model to investigate the $\mathrm{CSE}$ in $\mathrm{QC}_{2} \mathrm{D}$. Previous works using lattice QCD [27-30] and model calculations such as the Nambu-Jona-Lasinio model [39-42] and the low-energy effective theory based on the nonlinear realization $[25,26]$ imply the existence of the diquark condensed phase, or the diquark condensate phase, in a region of $\mu \geq m_{\pi} / 2$ ( $\mu$ is the quark chemical potential and $m_{\pi}$ is the pion mass) at zero temperature in $\mathrm{QC}_{2} \mathrm{D}$. The diquark condensed phase is described by $S$-wave, color-singlet, and flavor-singlet diquark condensate $\Delta \sim\left\langle\psi^{T} C \gamma_{5} \sigma^{2} \tau^{2} \psi\right\rangle$. Here, $\psi=\left(\psi_{u}, \psi_{d}\right)^{T}$ is a quark spinor in the two-color and two-flavor system, $C=i \gamma^{2} \gamma^{0}$ is the charge-conjugation operator, and $\sigma^{2}$ and $\tau^{2}$ are the antisymmetric Pauli matrices for color and flavor spaces, respectively.

In the present study, in order to incorporate effects from the diquark condensate but investigate the CSE in the most transparent way, we start with the following concise effective model,

\footnotetext{
${ }^{1}$ In contrast, the CS conductivity was found to be enhanced by heavy impurities in Refs. [37,38].
}

$$
\mathcal{L}_{\text {eff }}=\bar{\psi}\left(i \not D+\mu \gamma^{0}-m\right) \psi+\psi^{T} \Delta \psi
$$

In this Lagrangian a covariant derivative $D_{\mu} \psi=\left(\partial_{\mu}-\right.$ ie $\left.Q A_{\mu}\right) \psi$ with $Q=\operatorname{diag}\left(+\frac{2}{3},-\frac{1}{3}\right)$ the charge matrix has been included to describe interactions with the external magnetic field. $m$ is a quark mass and $\Delta=\gamma_{5} \sigma^{2} \tau^{2} \Delta$ is responsible for the diquark condensate. For convenience we rewrite Eq. (2) into the Nambu-Gorkov basis as

$$
\begin{aligned}
\mathcal{L}_{\text {eff }}= & \bar{\Psi}\left(\begin{array}{cc}
i \not \partial+\mu \gamma_{0}-m & \bar{\Delta} \\
\Delta & i \not \partial-\mu \gamma_{0}-m
\end{array}\right) \Psi \\
& +e \bar{\Psi}\left(\begin{array}{cc}
Q A & 0 \\
0 & -Q A
\end{array}\right) \Psi,
\end{aligned}
$$

where

$$
\Psi \equiv \frac{1}{\sqrt{2}}\left(\begin{array}{c}
\psi \\
\psi_{c}
\end{array}\right), \quad \bar{\Psi} \equiv \frac{1}{\sqrt{2}}\left(\bar{\psi}, \quad \bar{\psi}_{c}\right)
$$

are the Nambu-Gorkov spinors $\left(\psi_{c}=C \bar{\psi}^{T}\right)$. From the first line of Eq. (3), the inverse of the fermion propagator $S^{-1}\left(p_{0}, \boldsymbol{p}\right)$ reads as

$$
i S^{-1}\left(p_{0}, \boldsymbol{p}\right)=\left(\begin{array}{cc}
\not p+\mu \gamma^{0}-m & \overline{\boldsymbol{\Delta}} \\
\boldsymbol{\Delta} & \not p-\mu \gamma^{0}-m
\end{array}\right),
$$

with $\overline{\boldsymbol{\Delta}} \equiv \gamma^{0} \boldsymbol{\Delta}^{\dagger} \gamma^{0}$. By taking the inverse of $S^{-1}\left(p_{0}, \boldsymbol{p}\right)$, the fermion propagator is obtained as

$$
S\left(p_{0}, \boldsymbol{p}\right)=\left(\begin{array}{cc}
S_{11}\left(p_{0}, \boldsymbol{p}\right) & \sigma^{2} \tau^{2} S_{12}\left(p_{0}, \boldsymbol{p}\right) \\
\sigma^{2} \tau^{2} S_{21}\left(p_{0}, \boldsymbol{p}\right) & S_{22}\left(p_{0}, \boldsymbol{p}\right)
\end{array}\right),
$$

where each component has the Dirac structure as

$$
\begin{aligned}
& S_{11}\left(p_{0}, \boldsymbol{p}\right)=\sum_{\xi=\mathrm{p}, \mathrm{a}} S_{11}^{\xi}\left(p_{0}, \boldsymbol{p}\right) \Lambda_{\xi} \gamma^{0}, \\
& S_{12}\left(p_{0}, \boldsymbol{p}\right)=\sum_{\xi=\mathrm{p}, \mathrm{a}} S_{12}^{\xi}\left(p_{0}, \boldsymbol{p}\right) \Lambda_{\xi} \gamma_{5}, \\
& S_{21}\left(p_{0}, \boldsymbol{p}\right)=\sum_{\xi=\mathrm{p}, \mathrm{a}} S_{21}^{\xi}\left(p_{0}, \boldsymbol{p}\right) \Lambda_{\xi}^{C} \gamma_{5}, \\
& S_{22}\left(p_{0}, \boldsymbol{p}\right)=\sum_{\xi=\mathrm{p}, \mathrm{a}} S_{22}^{\xi}\left(p_{0}, \boldsymbol{p}\right) \Lambda_{\xi}^{C} \gamma^{0},
\end{aligned}
$$

with 


$$
\begin{aligned}
& S_{11}^{\xi}\left(p_{0}, \boldsymbol{p}\right)=i\left(\frac{\left|u_{\xi}(\boldsymbol{p})\right|^{2}}{p_{0}-\eta_{\xi} \epsilon_{\xi}(\boldsymbol{p})}+\frac{\left|v_{\xi}(\boldsymbol{p})\right|^{2}}{p_{0}+\eta_{\xi} \epsilon_{\xi}(\boldsymbol{p})}\right), \\
& S_{12}^{\xi}\left(p_{0}, \boldsymbol{p}\right)=-i\left(\frac{u_{\xi}^{*}(\boldsymbol{p}) v_{\xi}^{*}(\boldsymbol{p})}{p_{0}-\epsilon_{\xi}(\boldsymbol{p})}-\frac{u_{\xi}^{*}(\boldsymbol{p}) v_{\xi}^{*}(\boldsymbol{p})}{p_{0}+\epsilon_{\xi}(\boldsymbol{p})}\right), \\
& S_{21}^{\xi}\left(p_{0}, \boldsymbol{p}\right)=i\left(\frac{u_{\xi}(\boldsymbol{p}) v_{\xi}(\boldsymbol{p})}{p_{0}-\epsilon_{\xi}(\boldsymbol{p})}-\frac{u_{\xi}(\boldsymbol{p}) v_{\xi}(\boldsymbol{p})}{p_{0}+\epsilon_{\xi}(\boldsymbol{p})}\right), \\
& S_{22}^{\xi}\left(p_{0}, \boldsymbol{p}\right)=i\left(\frac{\left|v_{\xi}(\boldsymbol{p})\right|^{2}}{p_{0}-\eta_{\xi} \epsilon_{\xi}(\boldsymbol{p})}+\frac{\left|u_{\xi}(\boldsymbol{p})\right|^{2}}{p_{0}+\eta_{\xi} \epsilon_{\xi}(\boldsymbol{p})}\right) .
\end{aligned}
$$

Here we introduce $\eta_{\mathrm{p}}=+1$ and $\eta_{\mathrm{a}}=-1$. In these expressions, we defined the positive-energy and negative-energy projection operators $\Lambda_{\mathrm{p}}$ and $\Lambda_{\mathrm{a}}$ by

$$
\Lambda_{\xi}=\gamma_{0} \frac{E_{p} \gamma_{0}+\eta_{\xi}(m+\gamma \cdot p)}{2 E_{p}},
$$

with $E_{\boldsymbol{p}}=\sqrt{|\boldsymbol{p}|^{2}+m^{2}}$, and $\Lambda_{\mathrm{p}(\mathrm{a})}^{C}=\Lambda_{\mathrm{a}(\mathrm{p})}$, and

$$
\epsilon_{\xi}(\boldsymbol{p})=\sqrt{\left(E_{\boldsymbol{p}}-\eta_{\xi} \mu\right)^{2}+|\Delta|^{2}},
$$

are the dispersion relations for quasiparticles. The factors $u_{\mathrm{p}}(\boldsymbol{p}), v_{\mathrm{p}}(\boldsymbol{p}), u_{\mathrm{a}}(\boldsymbol{p})$, and $v_{\mathrm{a}}(\boldsymbol{p})$ satisfy relations

$$
\begin{aligned}
\left|u_{\xi}(\boldsymbol{p})\right|^{2} & =\frac{1}{2}\left(1+\frac{E_{\boldsymbol{p}}-\eta_{\xi} \mu}{\epsilon_{\xi}(\boldsymbol{p})}\right), \\
\left|v_{\xi}(\boldsymbol{p})\right|^{2} & =\frac{1}{2}\left(1-\frac{E_{\boldsymbol{p}}-\eta_{\xi} \mu}{\epsilon_{\xi}(\boldsymbol{p})}\right),
\end{aligned}
$$

and

$$
\left|u_{\xi}(\boldsymbol{p})\right|^{2}+\left|v_{\xi}(\boldsymbol{p})\right|^{2}=1, \quad u_{\xi}(\boldsymbol{p}) v_{\xi}(\boldsymbol{p})=\frac{\Delta}{2 \epsilon_{\xi}(\boldsymbol{p})} .
$$

By using the propagator of the quasiparticles in Eq. (6) together with Eqs. (7) and (8), and with the help of linear response theory, the CSE in the diquark condensed phase under a weak magnetic field can be evaluated.

\section{CS CONDUCTIVITY}

In the present work we investigate the CSE in the diquark condensed phase within linear response theory. Our calculations are similar to that for the chiral magnetic conductivity [36], but are more complicated due to the presence of diquark condensates. The axial current is defined by $j_{5}^{i}=\bar{\psi} \gamma^{i} \gamma_{5} \psi(i=1,2,3)$. In linear response theory we measure the difference between the current with and without external electromagnetic vector potential $A$,

$$
\left\langle j_{5}^{i}\right\rangle \equiv\left\langle j_{5}^{i}\right\rangle_{A}-\left\langle j_{5}^{i}\right\rangle_{A=0} .
$$

We note that the $U(1)_{A}$ axial current couples to the gluon topological density as $\partial^{\mu} j_{\mu}^{A} \sim \tilde{G}_{\mu \nu} G^{\mu \nu}$. Below we neglect the impact of $A$ on the color gauge dynamics, i.e., $\left\langle\tilde{G}_{\mu \nu} G^{\mu \nu}\right\rangle_{A}=\left\langle\tilde{G}_{\mu \nu} G^{\mu \nu}\right\rangle_{A=0}$. Then we focus on the anomalous contributions coupled to the $\mu$ and $A$.

In the following calculation, we will make use of the imaginary time formalism to incorporate finite temperature effects. In momentum space the axial current induced by the external electromagnetic field is evaluated as the retarded correlator between the axial vector and vector currents, $\left[\int_{p} \equiv \int d^{3} p /(2 \pi)^{3}\right]$

$$
\begin{aligned}
\left\langle j_{5}^{i}\left(i \bar{\omega}_{n}, \boldsymbol{q}\right)\right\rangle \equiv & \int_{0}^{\beta} d \tau \int d^{3} x\left\langle\bar{\psi}(x) \gamma^{i} \gamma_{5} \psi(x)\right\rangle \mathrm{e}^{-i \bar{\omega}_{n} \tau+i \boldsymbol{q} \cdot \boldsymbol{x}} \\
= & \frac{e}{2} \int_{\boldsymbol{p}} T \sum_{m} \operatorname{Tr}\left[\Gamma^{i} \Gamma_{5} S\left(i \omega_{m}, \boldsymbol{p}\right)\right. \\
& \left.\times\left(\Gamma_{\bar{\psi} A \psi}^{\mathrm{bare}}\right)^{\mu} S\left(i \omega_{m}^{\prime}, \boldsymbol{p}^{\prime}\right)\right] A_{\mu}\left(i \bar{\omega}_{n}, \boldsymbol{q}\right)
\end{aligned}
$$

with $\boldsymbol{p}^{\prime}=\boldsymbol{p}+\boldsymbol{q}, i \omega_{m}^{\prime}=i \omega_{m}+i \bar{\omega}_{n}$, and $N_{c}=2$, where we have defined the vertices in the Nambu-Gorkov space as

$\Gamma^{i} \Gamma_{5}=\left(\begin{array}{cc}\gamma^{i} \gamma_{5} & 0 \\ 0 & \gamma^{i} \gamma_{5}\end{array}\right), \quad\left(\Gamma_{\bar{\psi} A \psi}^{\mathrm{bare}}\right)^{\mu}=\left(\begin{array}{cc}Q \gamma^{\mu} & 0 \\ 0 & -Q \gamma^{\mu}\end{array}\right)$.

Here $\omega_{m}=(2 m+1) \pi T$ and $\bar{\omega}_{n}=2 n \pi T$ ( $n, m$ are integers) are the Matsubara frequencies for fermions and bosons, respectively. The symbol "Tr" stands for the trace with respect to the color, flavor, spinor, and Nambu-Gorkov spaces. (We note that, in the presence of symmetry breaking, the use of the bare vertices violates the conservation law and one must improve the vertices to recover it. We will come back to this point later but we are just mentioning that such improvements will not change the main conclusion of the simplest one loop results.)

We will leave only spatial components of the external gauge field $A_{\mu}\left(i \bar{\omega}_{n}, \boldsymbol{q}\right)$ because the magnetic field is solely generated by them. The real-time axial current $\left\langle j_{5}^{i}\left(q_{0}, \boldsymbol{q}\right)\right\rangle$ is given by the analytic continuation as

$$
\left\langle j_{5}^{i}\left(q_{0}, \boldsymbol{q}\right)\right\rangle=\left.\left\langle j_{5}^{i}\left(i \bar{\omega}_{n}, \boldsymbol{q}\right)\right\rangle\right|_{i \bar{\omega}_{n} \rightarrow q_{0}+i \eta},
$$

with $\eta$ an infinitesimal positive number.

Performing the trace with respect to the color, spinor, and Nambu-Gorkov spaces in Eq. (14), the axial current is reduced to the form

$$
\begin{aligned}
\left\langle j_{5}^{i}\left(i \bar{\omega}_{n}, \boldsymbol{q}\right)\right\rangle & =-i e \operatorname{tr}[Q] \epsilon^{i j k} A^{j}\left(i \bar{\omega}_{n}, \boldsymbol{q}\right) \sigma_{\mathrm{CSE}}^{k}\left(i \bar{\omega}_{n}, \boldsymbol{q}\right) \\
& =-e \operatorname{tr}[Q] B^{i} \sigma_{\mathrm{CSE}}\left(i \bar{\omega}_{n}, \boldsymbol{q}\right),
\end{aligned}
$$

where we used that $\sigma_{\mathrm{CSE}}^{k}\left(i \overline{\boldsymbol{\omega}}_{n}, \boldsymbol{q}\right)=q^{k} \sigma_{\mathrm{CSE}}\left(i \bar{\omega}_{n}, \boldsymbol{q}\right)$ because of the Lorentz structure, and $B^{i}=\epsilon^{i m n}\left(-i q^{m}\right) A^{n}$. Hence we can extract the conductivity $\sigma_{\mathrm{CSE}}\left(i \bar{\omega}_{n}, \boldsymbol{q}\right)$ as 


$$
\sigma_{\mathrm{CSE}}\left(i \bar{\omega}_{n}, \boldsymbol{q}\right)=\frac{q^{k}}{|\boldsymbol{q}|^{2}} \sigma_{\mathrm{CSE}}^{k}\left(i \bar{\omega}_{n}, \boldsymbol{q}\right)
$$

The function $\sigma_{\mathrm{CSE}}^{k}$ is computed using propagators decomposed into the particle and antiparticle pieces with normal and anomalous components, as in Eq. (7). Accordingly the function $\sigma_{\mathrm{CSE}}$ is also decomposed as

$$
\sigma_{\mathrm{CSE}}\left(i \bar{\omega}_{n}, \boldsymbol{q}\right)=\sum_{\xi, \xi^{\prime}=\mathrm{p}, \mathrm{a}} \sigma_{\mathrm{CSE}}^{\xi \xi^{\prime}}\left(i \bar{\omega}_{n}, \boldsymbol{q}\right)
$$

The set $\left(\xi, \xi^{\prime}\right)=(\mathrm{p}, \mathrm{p})$ and $(\mathrm{a}, \mathrm{a})$ correspond to the particlehole and antiparticle-antihole contributions, while (p, a) or $(a, p)$ are from the particle-antiparticle contributions. Explicitly,

$$
\sigma_{\mathrm{CSE}}^{\xi \xi^{\prime}}\left(i \bar{\omega}_{n}, \boldsymbol{q}\right)=\frac{N_{c} q^{k}}{|\boldsymbol{q}|^{2}} \int_{\boldsymbol{p}} \mathcal{F}^{\xi \xi^{\prime}}\left(\bar{\omega}_{n}, \boldsymbol{q} ; \boldsymbol{p}\right) \mathcal{K}_{\xi \xi^{\prime}}^{k}(\boldsymbol{q} ; \boldsymbol{p}),
$$

with the kinematic factor

$$
\mathcal{K}_{\xi \xi^{\prime}}^{k}(\boldsymbol{q} ; \boldsymbol{p})=-\eta_{\xi} \frac{p^{k}}{E_{\boldsymbol{p}}}+\eta_{\xi^{\prime}} \frac{\left(p^{\prime}\right)^{k}}{E_{\boldsymbol{p}^{\prime}}}
$$

which are independent of the diquark condensates, and the propagator part

$$
\begin{aligned}
\mathcal{F}^{\xi \xi^{\prime}}\left(\bar{\omega}_{n}, \boldsymbol{q} ; \boldsymbol{p}\right)= & T \sum_{m}\left[S_{11}^{\xi}\left(i \omega_{m}, \boldsymbol{p}\right) S_{11}^{\xi^{\prime}}\left(i \omega_{m}^{\prime}, \boldsymbol{p}^{\prime}\right)\right. \\
& -S_{12}^{\xi}\left(i \omega_{m}, \boldsymbol{p}\right) S_{21}^{\xi^{\prime}}\left(i \omega_{m}^{\prime}, \boldsymbol{p}^{\prime}\right) \\
& -S_{21}^{\xi}\left(i \omega_{m}, \boldsymbol{p}\right) S_{12}^{\xi^{\prime}}\left(i \omega_{m}^{\prime}, \boldsymbol{p}^{\prime}\right) \\
& \left.+S_{22}^{\xi}\left(i \omega_{m}, \boldsymbol{p}\right) S_{22}^{\xi^{\prime}}\left(i \omega_{m}^{\prime}, \boldsymbol{p}^{\prime}\right)\right]
\end{aligned}
$$

which carries the effects from the diquark condensates. In $\mathcal{F}^{\xi \xi^{\prime}}$ we can carry out the Matsubara summation using an identity

$$
T \sum_{m} \frac{1}{\left(i \omega_{m}-\epsilon_{1}\right)\left(i \omega_{m}^{\prime}-\epsilon_{2}\right)}=\frac{\tilde{f}\left(\epsilon_{1}\right)-\tilde{f}\left(\epsilon_{2}\right)}{i \bar{\omega}_{n}-\epsilon_{2}+\epsilon_{1}},
$$

where $\tilde{f}(\epsilon)=1 /\left(\mathrm{e}^{\beta \epsilon}+1\right)$ is the Fermi-Dirac distribution function. Using this identity, the function $\mathcal{F}^{\xi \xi^{\prime}}$ can be written as

$$
\begin{aligned}
\mathcal{F}^{\xi \xi^{\prime}}\left(\bar{\omega}_{n}, \boldsymbol{q} ; \boldsymbol{p}\right)= & \mathcal{C}_{1}^{\xi \xi^{\prime}}(\boldsymbol{q} ; \boldsymbol{p}) \mathcal{G}_{1}^{\xi \xi^{\prime}}(q ; p) \\
& +\mathcal{C}_{2}^{\xi \xi^{\prime}}(\boldsymbol{q} ; \boldsymbol{p}) \mathcal{G}_{2}^{\xi \xi^{\prime}}(q ; p),
\end{aligned}
$$

where the second term is nonzero only at finite temperature. The factors $\mathcal{C}^{\xi \xi^{\prime}}$ and $\tilde{\mathcal{C}}^{\xi \xi^{\prime}}$ are the coherence factors which carry the information about the wave functions $u_{\xi}$ and $v_{\xi}$,

$$
\begin{aligned}
& \mathcal{C}_{1,2}^{\mathrm{pp}}(\boldsymbol{q} ; \boldsymbol{p})=\frac{1}{2}\left[1 \mp \frac{\left.\left(E_{\boldsymbol{p}}-\mu\right)\left(E_{\boldsymbol{p}^{\prime}}-\mu\right)+|\Delta|^{2}\right]}{\epsilon_{\mathrm{p}}(\boldsymbol{p}) \epsilon_{\mathrm{p}}\left(\boldsymbol{p}^{\prime}\right)}\right], \\
& \mathcal{C}_{1,2}^{\mathrm{aa}}(\boldsymbol{q} ; \boldsymbol{p})=\frac{1}{2}\left[1 \mp \frac{\left(E_{\boldsymbol{p}}+\mu\right)\left(E_{\boldsymbol{p}^{\prime}}+\mu\right)+|\Delta|^{2}}{\epsilon_{\mathrm{a}}(\boldsymbol{p}) \epsilon_{\mathrm{a}}\left(\boldsymbol{p}^{\prime}\right)}\right], \\
& \mathcal{C}_{1,2}^{\mathrm{pa}}(\boldsymbol{q} ; \boldsymbol{p})=\frac{1}{2}\left[1 \pm \frac{\left(E_{\boldsymbol{p}}-\mu\right)\left(E_{\boldsymbol{p}^{\prime}}+\mu\right)-|\Delta|^{2}}{\epsilon_{\mathrm{p}}(\boldsymbol{p}) \epsilon_{\mathrm{a}}\left(\boldsymbol{p}^{\prime}\right)}\right], \\
& \mathcal{C}_{1,2}^{\mathrm{ap}}(\boldsymbol{q} ; \boldsymbol{p})=\frac{1}{2}\left[1 \pm \frac{\left(E_{\boldsymbol{p}}+\mu\right)\left(E_{\boldsymbol{p}^{\prime}}-\mu\right)-|\Delta|^{2}}{\epsilon_{\mathrm{a}}(\boldsymbol{p}) \epsilon_{\mathrm{p}}\left(\boldsymbol{p}^{\prime}\right)}\right]
\end{aligned}
$$

The upper (lower) sign in the right-hand side corresponds to the subscript 1 (2) in the left-hand side. The factors $\mathcal{G}_{1,2}^{\xi \xi^{\prime}}$ are the propagator factors which carry the information about the excitation spectra,

$$
\begin{aligned}
\mathcal{G}_{1}^{\xi \xi^{\prime}}(q ; p)= & \frac{1}{2}\left[1-\tilde{f}\left(\epsilon_{\xi}(\boldsymbol{p})\right)-\tilde{f}\left(\epsilon_{\xi^{\prime}}\left(\boldsymbol{p}^{\prime}\right)\right)\right] \\
& \times\left(\frac{1}{i \bar{\omega}_{n}+\epsilon_{\xi}(\boldsymbol{p})+\epsilon_{\xi^{\prime}}\left(\boldsymbol{p}^{\prime}\right)}\right. \\
& \left.+\frac{1}{-i \bar{\omega}_{n}+\epsilon_{\xi}(\boldsymbol{p})+\epsilon_{\xi^{\prime}}\left(\boldsymbol{p}^{\prime}\right)}\right),
\end{aligned}
$$

and

$$
\begin{aligned}
\mathcal{G}_{2}^{\xi \xi^{\prime}}(q ; p)= & -\frac{1}{2}\left[\tilde{f}\left(\epsilon_{\xi}(\boldsymbol{p})\right)-\tilde{f}\left(\epsilon_{\xi^{\prime}}\left(\boldsymbol{p}^{\prime}\right)\right)\right] \\
& \times\left(\frac{1}{i \bar{\omega}_{n}+\epsilon_{\xi}(\boldsymbol{p})-\epsilon_{\xi^{\prime}}\left(\boldsymbol{p}^{\prime}\right)}\right. \\
& \left.+\frac{1}{-i \bar{\omega}_{n}+\epsilon_{\xi}(\boldsymbol{p})-\epsilon_{\xi^{\prime}}\left(\boldsymbol{p}^{\prime}\right)}\right) .
\end{aligned}
$$

Below we examine how the contributions in different sectors affect the CSE conductivity.

\section{A. Particle-hole}

The coherence factors and propagators behave very differently for the normal and condensed phases. We consider the low temperature case $T \ll \Delta$ where quantum effects are important. The contributions which are most sensitive to the condensates are particle-hole contributions near the edge of the Fermi sea with $|\boldsymbol{p}| \simeq\left|\boldsymbol{p}^{\prime}\right| \simeq p_{F}$. Below we discuss the particle-hole contributions for the $|\boldsymbol{q}| \ll \Delta$ and $|\boldsymbol{q}| \gg \Delta$ cases. The latter can be seen as the results in the normal phase.

$$
\text { 1. }|\mathbf{q}| \ll \Delta
$$

Depending on the size of $\boldsymbol{q}$, the coherence factors appear constructively or destructively. To see this, first we set $\boldsymbol{q}=0$ as a special case of $|\boldsymbol{q}| \ll \Delta$. Then we find

$$
\mathcal{C}_{1}^{\mathrm{pp}}(\boldsymbol{q}=0 ; \boldsymbol{p})=0, \quad \mathcal{C}_{2}^{\mathrm{pp}}(\boldsymbol{q}=0 ; \boldsymbol{p})=1 .
$$


For $\mathcal{C}_{1}^{\mathrm{pp}}$, the correction starts with $O(\boldsymbol{q})$. With this $\mathcal{F}^{\mathrm{pp}} \sim$ $\mathcal{G}_{2}^{\mathrm{pp}}$ for small $\boldsymbol{q}$. For $\mathcal{G}_{2}^{\mathrm{pp}}$, we focus on $|\boldsymbol{p}| \simeq\left|\boldsymbol{p}^{\prime}\right| \simeq p_{F}$, where $\epsilon_{\mathrm{p}}\left(p_{F}\right)=\Delta$ and $\tilde{f} \sim \mathrm{e}^{-\Delta / T} \ll 1$. We expand

$$
\tilde{f}\left(\epsilon_{\mathrm{p}}(\boldsymbol{p})\right)-\tilde{f}\left(\epsilon_{\mathrm{p}}\left(\boldsymbol{p}^{\prime}\right)\right) \simeq-\left.\frac{\partial \tilde{f}(x)}{\partial x}\right|_{x=\epsilon_{\mathrm{p}}} \frac{\partial \epsilon_{\mathrm{p}}}{\partial \boldsymbol{p}} \cdot \boldsymbol{q},
$$

and get

$$
\left.\mathcal{G}_{2}^{\mathrm{pp}} \sim \frac{\partial \tilde{f}(x)}{\partial x}\right|_{x=\epsilon_{\mathrm{p}}} \frac{\left(\frac{\partial \epsilon_{\mathrm{p}}}{\partial \boldsymbol{p}} \cdot \boldsymbol{q}\right)^{2}}{\bar{\omega}_{n}^{2}+\left(\frac{\partial \epsilon_{\mathrm{p}}}{\partial \boldsymbol{p}} \cdot \boldsymbol{q}\right)^{2}} \sim \frac{1}{T} \mathrm{e}^{-\Delta / T} .
$$

For $T \rightarrow 0, \partial \tilde{f} / \partial x \rightarrow 0$ at $x>0$, and $\mathcal{G}_{2}^{\mathrm{pp}}$ is vanishing for $\Delta \neq 0$ as $\mathrm{e}^{-\Delta / T} / T \rightarrow 0$.

Finally we look at the kinematic factor coupling to $q^{k}$,

$$
\begin{aligned}
q^{k} \mathcal{K}_{\mathrm{pp}}^{k}(\boldsymbol{q} ; \boldsymbol{p}) & =-\frac{\boldsymbol{q} \cdot \boldsymbol{p}}{E_{\boldsymbol{p}}}+\frac{\boldsymbol{q} \cdot(\boldsymbol{p}+\boldsymbol{q})}{E_{\boldsymbol{p}+\boldsymbol{q}}} \\
& \simeq \frac{\boldsymbol{q}^{2}}{E_{\boldsymbol{p}}}-\frac{(\boldsymbol{p} \cdot \boldsymbol{q})^{2}}{E_{\boldsymbol{p}}^{3}} \sim \frac{\boldsymbol{q}^{2}}{E_{\boldsymbol{p}}}\left(1-\frac{1}{3} \frac{|\boldsymbol{p}|^{2}}{E_{\boldsymbol{p}}^{2}}\right),
\end{aligned}
$$

where in the last expression we averaged $(\boldsymbol{p} \cdot \boldsymbol{q})^{2} \rightarrow \boldsymbol{p}^{2} \boldsymbol{q}^{2} / 3$ in the integral over $\boldsymbol{p}$. Then the CSE conductivity from the particle-hole can be written as

$$
\sigma_{\mathrm{CSE}}^{\mathrm{pp}}\left(i \bar{\omega}_{n}, \boldsymbol{q}\right) \sim N_{c} \int_{\boldsymbol{p}} \mathcal{G}_{2}^{\mathrm{pp}} \frac{1}{E_{\boldsymbol{p}}}\left(1-\frac{1}{3} \frac{|\boldsymbol{p}|^{2}}{E_{\boldsymbol{p}}^{2}}\right),
$$

for small $\boldsymbol{q}$. Note that the thermal factor makes the expression Eq. (32) UV finite.

At zero temperature the particle-hole contributions vanish by the presence of the diquark gap as $\mathcal{G}_{2}^{\mathrm{pp}} \sim \mathrm{e}^{-\Delta / T} / T \rightarrow 0$. This suppression of particle-hole is what happens for the Meissner effect in a superconductor. In contrast, in a normal conductor the particle-hole induces the paramagnetic effects which cancel with the diamagnetic contributions from particle-antiparticle contributions. The absence of particle-hole contributions or paramagnetic effects, as found in our calculations for the diquark condensed phase, makes the material diamagnetic. The particle-hole pairs contribute only as thermal excitations.

\section{2. $|\mathbf{q}| \gg \Delta$ or normal phase}

For $|\boldsymbol{q}| \gg \Delta$, the above expression is qualitatively modified. Here it is important to note that $\left(E_{p}-\mu\right)$ $\left(E_{p^{\prime}}-\mu\right)<0$ for the particle-hole contributions; one of the excitations has $E>\mu$ and the other $E<\mu$. Then, neglecting $\Delta$, one finds

$$
\frac{\left(E_{\boldsymbol{p}}-\mu\right)\left(E_{\boldsymbol{p}^{\prime}}-\mu\right)+|\Delta|^{2}}{\epsilon_{\mathrm{p}}(\boldsymbol{p}) \epsilon_{\mathrm{p}}\left(\boldsymbol{p}^{\prime}\right)} \simeq-1,
$$

so that

$$
\mathcal{C}_{1}^{\mathrm{pp}}(\boldsymbol{q} ; \boldsymbol{p}) \simeq 1, \quad \mathcal{C}_{2}^{\mathrm{pp}}(\boldsymbol{q} ; \boldsymbol{p}) \simeq 0 .
$$

With this $\mathcal{F}^{\mathrm{pp}} \sim \mathcal{G}_{1}^{\mathrm{pp}}$ for $|\boldsymbol{q}| \gg \Delta$. This function is evaluated by focusing on the domain $|\boldsymbol{p}| \simeq\left|\boldsymbol{p}^{\prime}\right| \simeq p_{F}$. For $E_{\boldsymbol{p}}-$ $\mu \gg \Delta$ and $\mu-E_{p^{\prime}} \gg \Delta$, we use

$$
\begin{aligned}
1 & -\tilde{f}\left(\epsilon_{\mathrm{p}}(\boldsymbol{p})\right)-\tilde{f}\left(\epsilon_{\mathrm{p}}\left(\boldsymbol{p}^{\prime}\right)\right) \sim \tilde{f}\left(E_{\boldsymbol{p}^{\prime}}-\mu\right)-\tilde{f}\left(E_{\boldsymbol{p}}-\mu\right) \\
& \left.\sim \frac{\partial \tilde{f}(x)}{\partial x}\right|_{x=E_{\boldsymbol{p}}-\mu} \frac{\partial E_{\boldsymbol{p}}}{\partial \boldsymbol{p}} \cdot \boldsymbol{q}, \epsilon_{\xi}(\boldsymbol{p})+\epsilon_{\xi^{\prime}}\left(\boldsymbol{p}^{\prime}\right) \\
& \sim E_{\boldsymbol{p}}-E_{\boldsymbol{p}^{\prime}} \simeq-\frac{\partial E_{\boldsymbol{p}}}{\partial \boldsymbol{p}} \cdot \boldsymbol{q}
\end{aligned}
$$

to get

$$
\mathcal{G}_{1}^{\mathrm{pp}} \sim-\left.\frac{\partial \tilde{f}(x)}{\partial x}\right|_{x=E_{\boldsymbol{p}}-\mu} \times \frac{\left(\frac{\partial E_{\mathrm{p}}}{\partial \boldsymbol{p}} \cdot \boldsymbol{q}\right)^{2}}{\bar{\omega}_{n}^{2}+\left(\frac{\partial E_{\mathrm{p}}}{\partial \boldsymbol{p}} \cdot \boldsymbol{q}\right)^{2}} .
$$

For $T \rightarrow 0$, the contribution entirely comes from $|\boldsymbol{p}|=p_{F}$ where $\partial \tilde{f} / \partial x$ yields $-\delta\left(|\boldsymbol{p}|-p_{F}\right)$, as in the usual Debye screening calculations. Including the kinematic factor as before, we find

$$
\sigma_{\mathrm{CSE}}^{\mathrm{pp}}\left(i \bar{\omega}_{n}, \boldsymbol{q}\right) \sim N_{c} \int_{\boldsymbol{p}} \mathcal{G}_{1}^{\mathrm{pp}} \frac{1}{E_{\boldsymbol{p}}}\left(1-\frac{1}{3} \frac{|\boldsymbol{p}|^{2}}{E_{\boldsymbol{p}}^{2}}\right),
$$

for $|\boldsymbol{q}| \gg \Delta$. The expression Eq. (37) is UV finite and the integrand is dominated by the contributions from $|\boldsymbol{p}| \simeq p_{F}$.

As mentioned before, the result for $|\boldsymbol{q}| \gg \Delta$ can be taken as a result for the normal phase with $\Delta=0$. In the normal phase the particle-hole contributions are large at $T=0$ as they are gapless, in contrast to fermions in the diquark condensed phase. In the massless limit, we find, for the static limit,

$$
\left.\sigma_{\mathrm{CSE} ; m=0}^{\mathrm{pp}(\text { normal })}\right|_{q \rightarrow 0} ^{\omega=0} \rightarrow \frac{N_{c} \mu}{3 \pi^{2}},
$$

and for the dynamic limit

$$
\left.\sigma_{\mathrm{CSE} ; m=0}^{\mathrm{pp}(\text { normal })}\right|_{\boldsymbol{q}=0} ^{\omega \rightarrow 0} \rightarrow 0
$$

\section{B. Particle-antiparticle}

Next we discuss the particle-antiparticle contributions. These contributions are not regulated by thermal factors and are potentially UV divergent. Our main concern is the UV finiteness and for this purpose it is sufficient to study $T \sim 0$.

The coherence factors in the particle-antiparticle contributions are not dominated by the contributions from $|\boldsymbol{p}| \sim\left|\boldsymbol{p}^{\prime}\right| \simeq p_{F}$, but come from large phase space with momenta much larger than $\Delta$. For this reason we set 
$\Delta \rightarrow 0$ and focus on the leading order contributions. Then for $E_{p}-\mu>0$,

$$
\frac{\left(E_{\boldsymbol{p}}-\mu\right)\left(E_{\boldsymbol{p}^{\prime}}+\mu\right)-|\Delta|^{2}}{\epsilon_{\mathrm{p}}(\boldsymbol{p}) \epsilon_{\mathrm{a}}\left(\boldsymbol{p}^{\prime}\right)} \rightarrow 1,
$$

Accordingly,

$$
\mathcal{C}_{1}^{\mathrm{pa}}(\boldsymbol{q} ; \boldsymbol{p}) \rightarrow 1, \quad \mathcal{C}_{2}^{\mathrm{pa}}(\boldsymbol{q} ; \boldsymbol{p}) \rightarrow 0,
$$

and we find

$$
\mathcal{F}^{\mathrm{pa}} \rightarrow \mathcal{G}_{1}^{\mathrm{pa}}=\left[1-\tilde{f}\left(E_{\boldsymbol{p}}-\mu\right)-\tilde{f}\left(E_{\boldsymbol{p}^{\prime}}+\mu\right)\right] G_{\mathrm{pa}},
$$

where

$$
G_{\mathrm{pa}}=\frac{E_{p}+E_{p^{\prime}}}{\bar{\omega}_{n}^{2}+\left(E_{\boldsymbol{p}}+E_{p^{\prime}}\right)^{2}}
$$

Likewise,

$$
\mathcal{F}^{\text {ap }} \rightarrow \mathcal{G}_{1}^{\text {ap }}=\left[1-\tilde{f}\left(E_{\boldsymbol{p}}+\mu\right)-\tilde{f}\left(E_{\boldsymbol{p}^{\prime}}-\mu\right)\right] G_{\mathrm{pa}} .
$$

Meanwhile the kinematic factor is

$$
\mathcal{K}_{\mathrm{pa}}^{k}=-\mathcal{K}_{\mathrm{ap}}^{k}=-\left(\frac{p^{k}}{E_{\boldsymbol{p}}}+\frac{\left(p^{\prime}\right)^{k}}{E_{p^{\prime}}}\right) \sim-2 \frac{p^{k}}{E_{\boldsymbol{p}}},
$$

for small $\boldsymbol{q}$. While each of (pa) and (ap) contributions is UV divergent, the sum cancels the UV divergence.

$$
\begin{aligned}
\mathcal{F}^{\mathrm{pa}} & \mathcal{K}_{\mathrm{pa}}^{k}+\mathcal{F}^{\mathrm{ap}} \mathcal{K}_{\mathrm{ap}}^{k} \sim \mathcal{K}_{\mathrm{pa}}^{k}\left(\mathcal{F}^{\mathrm{pa}}-\mathcal{F}^{\mathrm{ap}}\right) \\
& \sim-2 \frac{p^{k}}{E_{\boldsymbol{p}}} G_{\mathrm{pa}}\left[\tilde{f}\left(E_{\boldsymbol{p}^{\prime}}-\mu\right)-\tilde{f}\left(E_{\boldsymbol{p}}-\mu\right)\right]-(\mu \leftrightarrow-\mu) \\
& \sim-\left.2 \frac{p^{k}}{E_{p}} \frac{(\boldsymbol{p} \cdot \boldsymbol{q})}{E_{\boldsymbol{p}}} G_{\mathrm{pa}} \sum_{\xi=\mathrm{p}, \mathrm{a}} \eta_{\xi} \frac{\partial \tilde{f}(x)}{\partial x}\right|_{x=E_{p}-\eta_{\xi} \mu} \\
& \sim-\left.\frac{2 q^{k}}{3} \frac{\boldsymbol{p}^{2}}{E_{p}^{2}} G_{\mathrm{pa}} \sum_{\xi=\mathrm{p}, \mathrm{a}} \eta_{\xi} \frac{\partial \tilde{f}(x)}{\partial x}\right|_{x=E_{p}-\eta_{\xi} \mu},
\end{aligned}
$$

where we took the angular average for $\boldsymbol{p}$. The CS conductivity is

$$
\sigma_{\mathrm{CSE}}^{\mathrm{pa}+\mathrm{ap}}\left(i \bar{\omega}_{n}, \boldsymbol{q}\right) \sim-\left.N_{c} \int_{\boldsymbol{p}} \frac{2}{3} \frac{\boldsymbol{p}^{2}}{E_{\boldsymbol{p}}^{2}} G_{\mathrm{pa}} \sum_{\xi=\mathrm{p}, \mathrm{a}} \eta_{\xi} \frac{\partial \tilde{f}(x)}{\partial x}\right|_{x=E_{p}-\eta_{\xi} \mu} .
$$

Because of $\partial \tilde{f} / \partial x$, the integrand is dominated by states with $E_{p} \pm \mu$. In the massless limit, we find, for the static limit,

$$
\left.\sigma_{\mathrm{CSE} ; m=0}^{\mathrm{pp}(\text { normal })}\right|_{q \rightarrow 0} ^{\omega=0} \rightarrow \frac{N_{c} \mu}{6 \pi^{2}}
$$

and for the dynamic limit

$$
\left.\sigma_{\mathrm{CSE} ; m=0}^{\mathrm{pp}(\mathrm{normal})}\right|_{q=0} ^{\omega \rightarrow 0} \rightarrow \frac{N_{c} \mu}{6 \pi^{2}} .
$$

That is, the static and dynamic limits are the same, as the propagators do not have sensitivity to the limiting order.

\section{Antiparticle-antihole}

The computations of antiparticle-antihole proceed in the very similar way as the particle-hole case. The contributions are suppressed by thermal factor as $\sim \mathrm{e}^{-(\mu+\Delta) / T}$.

\section{NUMERICAL RESULTS}

In this section, we present the numerical results of the CS conductivity for plural density, quark mass, and temperature. We discuss a normalized CS conductivity,

$$
R^{\xi \xi^{\prime}} \equiv \sigma_{\mathrm{CSE}}^{\xi \xi^{\prime}} / \sigma_{0}, \quad R=\sum_{\xi, \xi^{\prime}=\mathrm{p}, \mathrm{a}} R^{\xi \xi^{\prime}}
$$

with a $\mu$ dependent normalization factor

$$
\sigma_{0}=\frac{N_{c} \mu}{2 \pi^{2}},
$$

where $\sigma_{0}$ is the conductivity at massless, zero temperature, and static limits in the normal phase.

To understand the results in this section, it is useful to use the massless and zero temperature limit in the normal phase as a baseline. The static conductivity is

$$
\left(R^{\mathrm{pp}}+R^{\mathrm{pa}+\mathrm{ap}}+R^{\mathrm{aa}}\right)_{\substack{\omega=0 \\ q \rightarrow 0}}^{\omega=0} \frac{2}{3}+\frac{1}{3}+0=1,
$$

and the dynamic conductivity is

$$
\left(R^{\mathrm{pp}}+R^{\mathrm{pa}+\mathrm{ap}}+R^{\mathrm{aa}}\right)_{q=0}^{\omega \rightarrow 0} \rightarrow 0+\frac{1}{3}+0=\frac{1}{3} .
$$

The difference comes from the response of particle-hole to the external fields.

To delineate the CS conductivity we found it convenient to discuss the normal phase as a guideline. We discuss the CS conductivity including the mass effects, $\mu$ dependence, and temperature dependence. Finally we include the diquark gap. We focus on the $\omega=0$ case; the impact of $\omega$ can be readily seen from the propagator factors.

In this section we present the CS conductivity pretending that quark matter exists from $\mu \geq m$. This picture is not realistic at $\mu \sim m$ for $\mathrm{QC}_{2} \mathrm{D}$ where a hadronic phase should exist at $\mu<m_{\pi} / 2$ and diquarks with the mass $m_{\pi}$ condense at $\mu \geq m_{\pi} / 2$. At low density there are more appropriate presentations based on the chiral Lagrangian [43], and we will not repeat those discussions. Hence, only our results at $\mu \gg m$ can be taken at its face value. Here the chiral 
symmetry is supposed to get restored and $m$ will be regarded as the current quark mass. As for comparisons with the lattice simulation setup, we note that the pion mass used is about $700 \mathrm{MeV}$, and we should regard the current quark mass as rather heavy. We take $m \sim 100 \mathrm{MeV}$ as a typical value.

\section{A. Normal phase}

First we examine the current quark mass dependence. A finite current quark mass breaks the chiral symmetry explicitly, blurring qualitative pictures for chiral conductivities whose descriptions are usually based on massless quarks. Whether the mass effects enter as a quark mass or the mass of NG modes depend on the phase structure of the $\mathrm{QC}_{2} \mathrm{D}$, but we consider the former case as a guide for the domain of $\mu \gg m$. Shown in Fig. 1 is the (normalized) static CS conductivity at zero temperature. It sets the overall size. The cases with various quark masses $m$ are displayed. Clearly the mass characterizes the onset of quark density. We have checked the scaling

$$
\left.\sigma_{\mathrm{CSE}}\right|_{q \rightarrow 0} ^{\omega=0}=\frac{N_{c} \sqrt{\mu^{2}-m^{2}}}{2 \pi^{2}}=\frac{N_{c} p_{F}}{2 \pi^{2}},
$$

where $p_{F}$ is the Fermi momentum in the normal phase, related to the baryon density as $p_{F}=\left(3 \pi^{2} n_{B} / N_{f}\right)^{1 / 3}$. This expression is useful to understand that the CS conductivity is sensitive to the number density, rather than the chemical potential. Below we set $m=0$.

Next we examine the $\mu$ dependence of the normalized CS conductivity $R$ at various external momenta $|\boldsymbol{q}|$, shown in Fig. 2. The $R$ in low momentum limit approaches 1 but damps at larger momenta. Its size becomes half at $|\boldsymbol{q}|=2 \mu=2 p_{F}$, but after that the damping proceeds rather slowly.

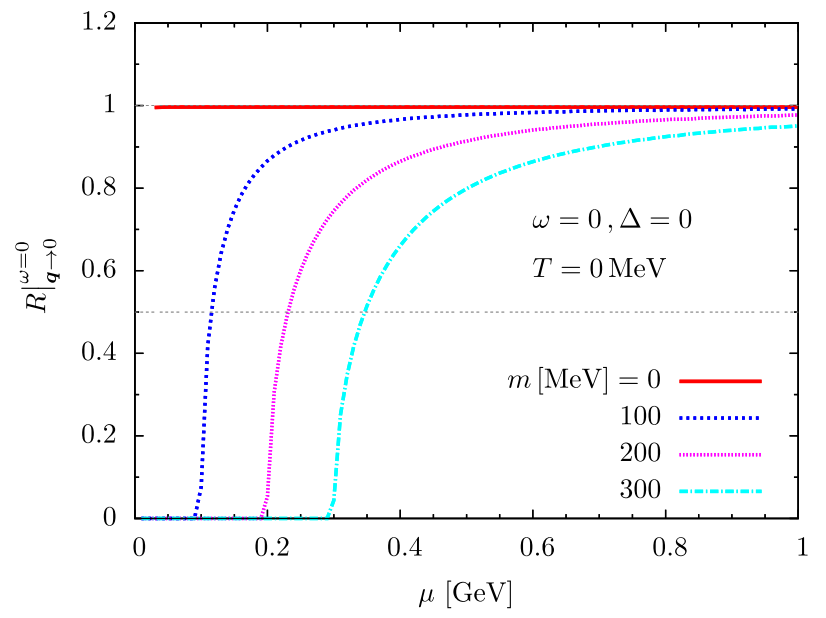

FIG. 1. The $\mu$ dependence of the CS conductivity for the quark masses $m=0,100,200$, and $300 \mathrm{MeV}$, where $\omega, T, \Delta$ are set to zero.



FIG. 2. The $\mu$ dependence of the CS conductivity as a function of spatial momenta $\boldsymbol{q}$. As an eye guide we show a $R=0.5$ line which is found at $|\boldsymbol{q}|=2 \mu=2 p_{F}$.

Shown in Fig. 3 is the temperature dependence of $R$. We fixed $\mu=500 \mathrm{MeV}$. The $R$ at $\boldsymbol{q} \rightarrow 0$ limit stays constant at a larger temperature. The growth is more evident at high momentum modes.

\section{B. Diquark condensed phase: Schematic setup}

Now we turn on the diquark gap. The major roles of $\Delta$ is to distinguish the regimes, $|\boldsymbol{q}|<\Delta$ and $|\boldsymbol{q}|>\Delta$, and to determine the abundance of thermal quarks which is controlled by a thermal factor $\tilde{f} \sim \mathrm{e}^{-\Delta / T}$. Its impact is substantial only for the low-momentum or low-temperature behaviors, $|\boldsymbol{q}|<\Delta$ or $T<\Delta$. In the other domains the results are similar to the normal phase.

Shown in Fig. 4 is the $\Delta$ dependence of the static CS conductivity as a function of spatial momenta $\boldsymbol{q}$. The values of $\Delta$ are $0,1,10,50,100,150$, and $200 \mathrm{MeV}$. We set $T=m=\omega=0$ and $\mu=500 \mathrm{MeV}$. It is clear that the size

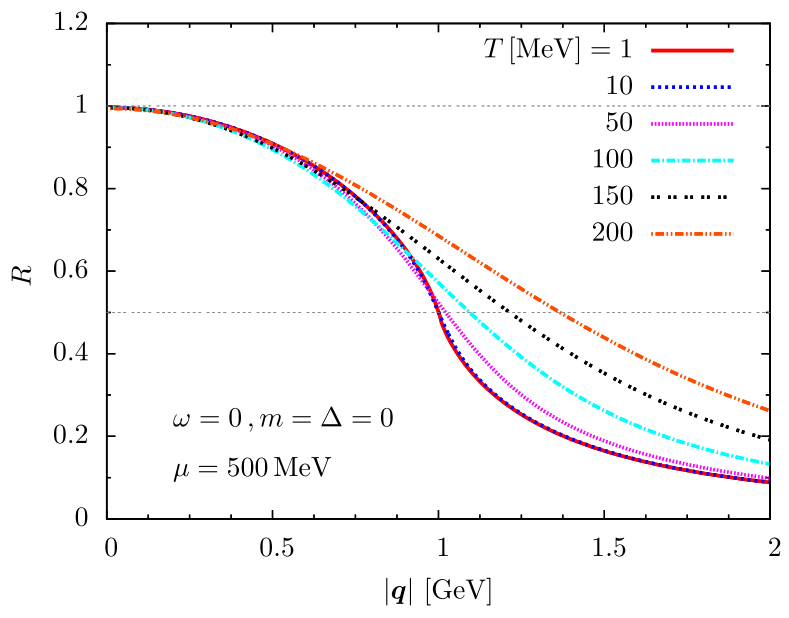

FIG. 3. The $T$ dependence of the CS conductivity as a function of spatial momenta $\boldsymbol{q}$. 


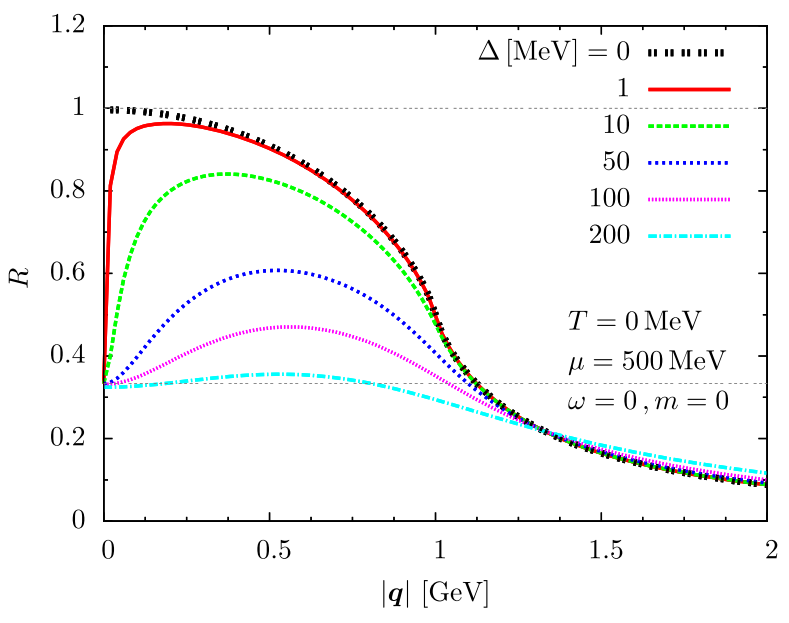

FIG. 4. The $\Delta$ dependence of the CS conductivity. We set $T=m=\omega=0$ and $\mu=500 \mathrm{MeV}$. The values of $\Delta$ are 0,1 , 10, 50, 100, and $200 \mathrm{MeV}$. We show $R=1 / 3$ and 1 for eye guides.

of $\Delta$ determines the domain of $\boldsymbol{q}$ where the CS conductivity deviates from that in the normal phase. With a finite $\Delta$, the static conductivity is $R=1 / 3$, reflecting the suppression of the particle-hole contributions by diquark gaps.

The temperature dependence of the CS conductivity can be also understood by the suppression of particle-holes. Shown in Fig. 5 is the temperature dependence of the static CS conductivity at a fixed gap, $\Delta=200 \mathrm{MeV}$ (more realistic treatments of $\Delta$ will be discussed in the next section). At low temperature $R=1 / 3$; as the temperature increases from $T=0$ to $T \simeq \Delta$, thermal quarks contribute as in the normal phase, and $R$ increases from $R=1 / 3$ to the value $R=1$ of the normal phase.

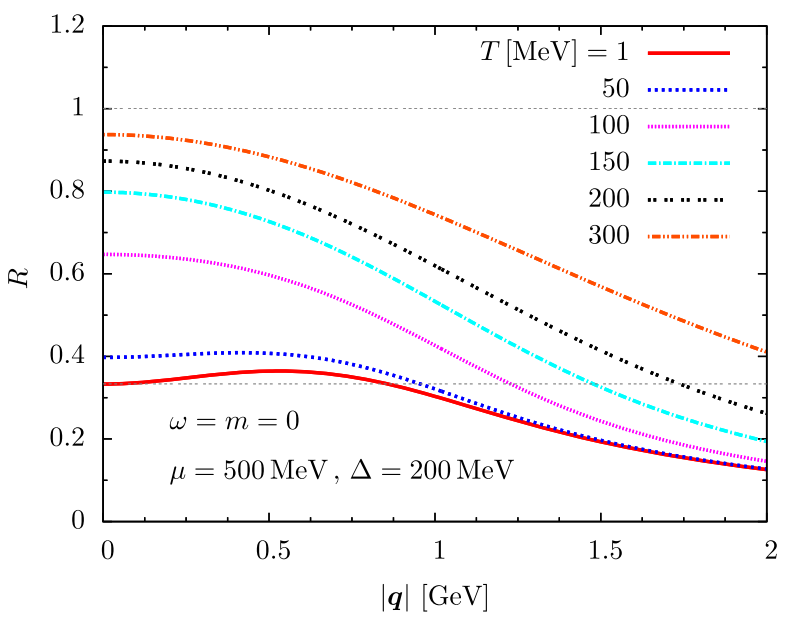

FIG. 5. The $T$ dependence of the CS conductivity. We set $m=\omega=0, \mu=500 \mathrm{MeV}$, and $\Delta=200 \mathrm{MeV}$. The values of $T$ are $1,50,100,150$, and $200 \mathrm{MeV}$.

\section{Diquark condensed phase: Realistic setup}

Finally we consider a parameter set consistent with lattice simulations for $\mathrm{QC}_{2} \mathrm{D}$, and consider the static CS conductivity at $\omega=0$ and $\boldsymbol{q} \rightarrow 0$ for various $\mu$ and $T$. Most of the lattice simulations have been done for relatively heavy pion mass $\simeq 700 \mathrm{MeV}$ and $\rho$ meson mass of $\sim 900 \mathrm{MeV}$. The onset chemical potential $\mu_{c}$ for the baryon density is $\mu_{c}=m_{\pi} / 2 \simeq 350 \mathrm{MeV}$. For comparisons of our analytic results with the future lattice simulations in the diquark condensed phase, we use a relatively large current quark mass of $m=100 \mathrm{MeV}$ as in our previous works [31,32], and assume $m_{\pi}=700 \mathrm{MeV}$ in this setup.

Also, at this stage we introduce the $\mu$ and $T$ dependence of the gap. For the $\mu$ dependence, we assume the zero temperature gap of the form [25]

$$
\Delta_{0}(\mu)=\bar{\Delta}_{0} \sqrt{1-\left(\mu_{c} / \mu\right)^{4}} \theta\left(\mu-\mu_{c}\right),
$$

as predicted by the chiral effective theories. The $\Delta_{0}$ vanishes at $\mu=\mu_{c}$, while approaches $\bar{\Delta}_{0}$ at high density. As for the temperature dependence, we assume the Bardeen-Cooper-Schrieffer (BCS) formulas as our baseline [44]

$$
T_{\mathrm{SF}}(\mu) \simeq 0.57 \Delta_{0}(\mu),
$$

where $T_{\mathrm{SF}}(\mu)$ is the critical temperature of the diquark condensed phase at given $\mu$. The gap depends on $T$ as

$$
\Delta(\mu, T) \simeq \Delta_{0}(\mu)\left(1-T / T_{\mathrm{SF}}\right)^{1 / 2} .
$$

For the high density value of the diquark gap, we take $\bar{\Delta}_{0}=200 \mathrm{MeV}$ which, according to the BCS formulas, leads to $T_{\mathrm{SF}} \simeq 114 \mathrm{MeV}$. This value is consistent with the lattice results $T_{\mathrm{SF}}=90-120 \mathrm{MeV}$ in the BCS domain of $\mathrm{QC}_{2} \mathrm{D}$ matter for $\mu \gtrsim 500 \mathrm{MeV}$, where the lattice results showed that $T_{\mathrm{SF}}$ depends on $\mu$ weakly. See Ref. [31] for more detailed considerations on the applicability of the formulas.

Shown in Fig. 6 is the static CS conductivity for various $T$ and $\mu$. We display results only for $\mu \geq \mu_{c}$ and $T<T_{\mathrm{SF}}$; at lower $\mu$ and $T$ we should use the hadronic degrees of freedom. The normalized CS conductivity $R$ is close to $1 / 3$ at low temperature and approaches $R=1$ at higher temperatures as expected for the normal phase. The growth of $R$ is much quicker than in Fig. 5 because now we are including the temperature dependence of gaps. For the same reason, the rapid change in $R$ at low $\mu$ happens because the $T_{\mathrm{SF}}$ is low, accordingly $\Delta$ is smaller and thermal quarks can more easily contribute to the conductivity.

We emphasize that our results on the temperature dependence are based on the assumption that thermal quarks behave as in an ideal gas of (quasi)particles; that is, once particles and holes are released from diquark condensates, they 


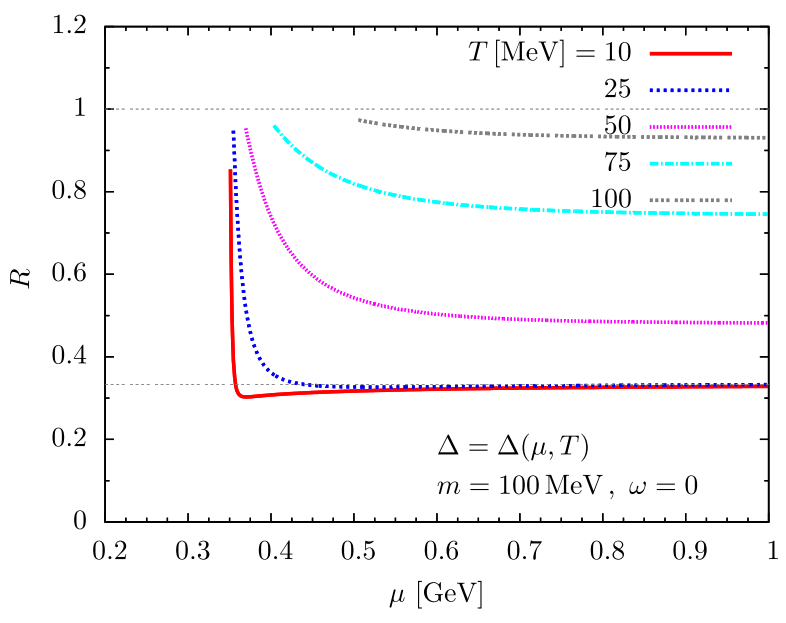

FIG. 6. The $T$ and $\mu$ dependence of the static CS conductivity with $\Delta$ prepared for comparisons with the lattice simulations. We terminate the lines for low $\mu$ as our descriptions based on quarks are valid only for $\mu \geq \mu_{c}$ and $T<T_{\mathrm{SF}}$. The low density and temperature domain should be calculated with hadronic degrees of freedom.

behave individually. But the validity of this picture is not immediately obvious except at very high density. The thermally excited particles and holes may need to form hadronic objects to avoid the energetic cost of the color electric flux from isolated quarks. If this is the case, the thermal factor necessary for excitations are $\sim \mathrm{e}^{-2 \Delta / T}$ of a particle-hole pair, instead of $\sim \mathrm{e}^{-\Delta / T}$ for an isolated quark [31]; the thermal corrections would be much smaller than we computed. In this respect the lattice simulations in diquark condensed phase at low density are important to delineate the properties of thermal excitations in dense matter.

\section{DISCUSSIONS}

Our calculations of the CS conductivity rely on the validity of quasiparticle pictures in the diquark condensed phase. While our results on the normal phase lead to the static CS conductivity at $\boldsymbol{q} \rightarrow 0$ limit as expected from the anomaly arguments, our calculations for the diquark condensed phase lead to only the one-third; the coefficient of the CSE is not universal even in chiral limit.

This conclusion puzzled us. The triangle graph for the A-V-V type (A: axial-vector, $\mathrm{V}$ : vector) arises when we expand the A-V correlator by the term $\mu \gamma_{0}$ in the quark propagators as $S \simeq S_{0}+S_{0}\left(-\mu \gamma_{0}\right) S_{0}+\cdots$ where $S_{0}$ is the propagator at $\mu=0$. However, it turned out that such expansion does not yield the same result as the usual triangle calculations. In fact, the static CS conductivity at vanishing temperature in the diquark condensed phase can be expanded with respect to $\mu$ as

$\left.\sigma_{\mathrm{CSE}}\right|_{q \rightarrow 0} ^{\omega=0}=\frac{N_{c}}{6 \pi^{2}} \frac{|\Delta|^{2}}{m^{2}+|\Delta|^{2}} \mu+\mathcal{O}\left(\mu^{2}\right) \quad$ at $T=0$.
Here we have checked that the higher-order contributions $\mathcal{O}\left(\mu^{2}\right)$ are proportional to $|\Delta|^{2} m^{2}$. In this way, the conductivity is shown to be given by $S_{0}\left(-\mu \gamma_{0}\right) S_{0}$ part solely in chiral limit $(m \rightarrow 0)$, resulting in

$$
\left.\sigma_{\mathrm{CSE}}\right|_{q \rightarrow 0} ^{\omega=0} \rightarrow \frac{C_{\text {uiv. }}}{3} \mu
$$

with $C_{\text {univ. }}=N_{c} /\left(2 \pi^{2}\right)$ the universal value. It should be noted that, in Eq. (58), the dimensionless expansion parameter is found to be $\mu / \sqrt{|\Delta|^{2}+m^{2}}$, hence $m \rightarrow 0$ limit to obtain (59) and has been safely taken due to a regulator played by $\Delta$. On the other hand, the other limit $\Delta \rightarrow 0$ in Eq. (58) leads to an incorrect answer; $\left.\sigma_{\mathrm{CSE}}\right|_{q \rightarrow 0} ^{\omega=0} \rightarrow 0$, showing the diquark gap $\Delta$ changes the analytic properties of the conductivity in the infrared.

The above violation of the universality does not seems to be unnatural to us. The $\mu \gamma_{0}$ vertex in $S_{0}\left(-\mu \gamma_{0}\right) S_{0}$ introduces no momenta; the only available momentum, after integrating out the loop momenta, is the external momentum $q$. Thus the possible form from the expansion of the A-V correlator in obtaining Eq. (59) should be

$$
\left\langle j_{5}^{i}(q) j_{e m}^{j}(-q) j_{B}^{0}(0)\right\rangle_{A=0}^{\mu=0} \mu A_{j} \sim \epsilon^{0 i j k} \mu q^{j} A^{k} .
$$

Contracting with the external momentum $q^{i}$, the spatial divergence of axial current vanishes, showing that our results do not affect $\partial_{\mu} j_{5}^{\mu}$ and hence the anomaly relation. From this viewpoint it is not clear to us why the CS coefficient should be constrained by the anomaly. The possible exceptions for this discussion are the cases when $A^{k}$ is singular $[45,46]$, as in the Dirac string on which $\left(\partial_{i} \partial_{j}-\partial_{j} \partial_{i}\right) \boldsymbol{A} \neq 0$, but this sort of external fields are presumably not compatible with the linear response regime utilized in this paper. The radiative corrections to chiral transport coefficients have been discussed in Refs. [47-49].

On the other hand, our one-loop calculations shown in the previous sections have not taken into account the vertex correction, and hence would miss some qualitatively important effects. In particular, we must treat the quasiparticle propagators and the vertices consistently in order to keep the conservation laws. Our quasiparticle propagators include diquark mean fields which break the $U(1)_{A}$ axial, baryon number, and electric charge conservations. The improved vertices produce the poles of the NG modes that recover the conservation laws.

Below we give a brief discussion on the structure of the improved vertices and will conclude that they do not influence the conclusions in the simplest quasiparticle computations.

The vertex corrections appear both in the axial-vector current and the vector current coupled to background electromagnetic fields. The WTI for the axial-vector vertex leads to 


$$
q^{\mu} \overline{\boldsymbol{\Gamma}}_{\mu}^{A}-2 m \Gamma_{5}=i S^{-1}(p+q) \gamma_{5}+\gamma_{5} i S^{-1}(p),
$$

where the improved vertex is the sum of the bare and the correction, $\overline{\boldsymbol{\Gamma}}_{\mu}^{A}=\left(\Gamma_{\text {bare }}\right)_{\mu}^{5}+\delta \Gamma_{\mu}^{A}$, with

$$
q^{\mu} \delta \Gamma_{\mu}^{A}=2\left(\begin{array}{cc}
0 & \overline{\boldsymbol{\Delta}} \\
\boldsymbol{\Delta} & 0
\end{array}\right)=Z_{A} .
$$

[Reminder: $\boldsymbol{\Delta}=\gamma_{5} \sigma^{2} \tau^{2} \Delta$ and $\overline{\boldsymbol{\Delta}}=-\gamma_{5} \sigma^{2} \tau^{2} \Delta^{*}$.] Similarly, the WTI with respect to the $U(1)_{\mathrm{em}}$ gauge symmetry leads to

$$
q^{\mu} \overline{\boldsymbol{\Gamma}}_{\mu}^{V}=\mathcal{Q} i S^{-1}(p+q)-i S^{-1}(p) \mathcal{Q},
$$

where $\mathcal{Q}=\operatorname{diag}(Q,-Q)$ and $\overline{\boldsymbol{\Gamma}}_{\mu}^{V}=\Gamma_{\mu}^{\mathrm{bare}}+\delta \Gamma_{\mu}^{V}$ with

$$
q^{\mu} \delta \Gamma_{\mu}^{V}=\left(\begin{array}{cc}
0 & Q \overline{\boldsymbol{\Delta}}+\overline{\boldsymbol{\Delta}} Q \\
-Q \boldsymbol{\Delta}-\boldsymbol{\Delta} Q & 0
\end{array}\right)=Z_{V} .
$$

For Eqs. (62) and (64) to be valid for $q \rightarrow 0$, the correction of the vertices $\left(\delta \Gamma_{\mu}^{A}, \delta \Gamma_{\mu}^{V}\right)$ must contains poles; otherwise the left-hand side would vanish in spite of the finite righthand side. The general form of solutions is (assuming the linear dispersion between $q_{0}$ and $\boldsymbol{q}$ )

$$
\delta \Gamma_{\mu}^{s}=\frac{\delta^{\mu 0} q_{0}+v_{s} \delta^{i \mu} q_{i}}{q_{0}^{2}-v_{s}^{2} q^{2}} Z_{s}
$$

where $s=V, A$, and $v_{s}$ is the medium velocity of the NG mode. From this expression it is clear the corrections to the vertices are proportional to the external momenta $\boldsymbol{q}$. Therefore, it yields a term of the form $\sim \epsilon^{0 i j k} q_{i} q_{j} A_{k}$, and drops off from the evaluation of $\left\langle j_{5}^{i}\right\rangle$ for a regular $A_{k}$. Thus, while the NG modes join the anomalous processes, they do not contribute to the coefficients of the anomaly relations.

With these discussions, it seems plausible to us that the CS conductivity in chiral limit depends on the phase structure. We note that our calculations correspond to the bulk part of matter. If the coefficient of the CSE is indeed universal, it likely requires the manifest treatments of the boundaries of matter which in turn introduce space variation of the chemical potential. We expect that such boundaries accommodate surface zero modes that contribute to the CS conductance as a global quantity, rather than the conductivity as a local quantity.

\section{CONCLUSIONS}

In this paper we have delineated the CS conductivity within the quasiparticle picture. The CS conductivity has been calculated in lattice simulations but the result was for the domain other than the diquark condensed phase [34].
We hope that our considerations in this paper will be tested in near future.

The results depend on the particle-hole contributions which are sensitive to the phase structure of $\mathrm{QC}_{2} \mathrm{D}$. The particle-hole contributions are suppressed in the presence of diquark condensates. As a consequence, the static CS conductivity at a low-momentum limit leads to only the one-third of the normal phase. We note that this particlehole suppression is also the origin of the electromagnetic Meissner mass, relating the CS conductivity to the Meissner effects. As the temperature increases, the particles-holes come back to enhance the CS conductivity to the size of the normal phase.

In a more general context, the nature of thermal corrections carry important information on the properties of matter other than the diquark condensates. Thermal excitations on top of the diquark condensed Fermi surface may be hadronic. This sort of picture has been suggested in the quarkyonic matter conjecture where the quark matter has the baryonic Fermi surface [50-54]. We studied this point of view by examining gluon propagators in the diquark condensed phase [31], and found indications that thermal quarks, rather than thermal hadrons, induce too strong thermal corrections to gluons. In order to derive definite conclusions, however, we need more lattice data points at low temperatures and should also examine the systematics in our calculations. The CS conductivity may provide us information complementary with those from gluon propagators.

In the astrophysical aspect, the understandings of excitations in dense $\mathrm{QC}_{2} \mathrm{D}$ have important applications to the physics of neutron stars [55]. In this context, recently the picture of quark-hadron continuity has been actively discussed $[23,56-65])$ to account for the interplay between the low-density nuclear physics [66], the neutron star radii for 1.4- $[67,68]$ and 2-solar mass $\left(M_{\odot}\right)$ neutron stars [69-72], and the maximum mass $\simeq 2.08 \pm 0.07 M_{\odot}$ [73]. In particular the latest result by NICER [69-72] shows that the radii of $1.4 M_{\odot}$ and $2 M_{\odot}$ neutron stars are close $(12-13 \mathrm{~km}$ for both), disfavoring strong first-order transitions from the nuclear to quark matter domain, and implying that there should be rather stiff matter with the sound velocity exceeding the conformal value, ${ }^{2} \sqrt{1 / 3}[23,74-78]$. These continuity discussions are basically for neutron star matter at zero temperature. In order to expand them into the level of finite temperature and general charge chemical potentials, we need to have more detailed insights on the excitations in dense QCD [79]. $\mathrm{QC}_{2} \mathrm{D}$ is an ideal laboratory to delineate these issues and further studies are called for.

\footnotetext{
${ }^{2}$ To the best of our knowledge, this behavior first appeared in Ref. [74,75] in the context of quark-hadron crossover model. A more general argument based on nuclear physics and neutron star observations was given in Ref. [76], and a microscopic description was proposed in Ref. [77] in the quarkyonic matter context.
} 


\section{ACKNOWLEDGMENTS}

D. S. wishes to thank Naoki Yamamoto for useful comments. Also, the authors thank Yoshimasa Hidaka and Noriyuki Sogabe for fruitful discussions and comments. T. K. is supported by NSFC Grant No. 11875144 .
[1] S. L. Adler, Axial vector vertex in spinor electrodynamics, Phys. Rev. 177, 2426 (1969).

[2] J. S. Bell and R. Jackiw, A PCAC puzzle: $\pi^{0} \rightarrow \gamma \gamma$ in the $\sigma$ model, Nuovo Cimento A 60, 47 (1969).

[3] L. Alvarez-Gaume and E. Witten, Gravitational anomalies, Nucl. Phys. B234, 269 (1984).

[4] A. Vilenkin, Equilibrium parity violating current in a magnetic field, Phys. Rev. D 22, 3080 (1980).

[5] D. E. Kharzeev, L. D. McLerran, and H. J. Warringa, The effects of topological charge change in heavy ion collisions: 'Event by event $\mathrm{P}$ and $C P$ violation', Nucl. Phys. A803, 227 (2008).

[6] K. Fukushima, D. E. Kharzeev, and H. J. Warringa, The chiral magnetic effect, Phys. Rev. D 78, 074033 (2008).

[7] D. T. Son and P. Surowka, Hydrodynamics with Triangle Anomalies, Phys. Rev. Lett. 103, 191601 (2009).

[8] D. Thanh Son and N. Yamamoto, Berry Curvature, Triangle Anomalies, and the Chiral Magnetic Effect in Fermi Liquids, Phys. Rev. Lett. 109, 181602 (2012).

[9] M. A. Stephanov and Y. Yin, Chiral Kinetic Theory, Phys. Rev. Lett. 109, 162001 (2012).

[10] N. Yamamoto, Chiral transport of neutrinos in supernovae: Neutrino-induced fluid helicity and helical plasma instability, Phys. Rev. D 93, 065017 (2016).

[11] S. L. Adler and W. A. Bardeen, Absence of higher order corrections in the anomalous axial vector divergence equation, Phys. Rev. 182, 1517 (1969).

[12] S. L. Adler, Anomalies to all orders, in 50 Years of YangMills Theory, edited by G. 't Hooft (World Scientific Publishing, Hackensack, NJ, 2005).

[13] S. Golkar and D. T. Son, (Non)-renormalization of the chiral vortical effect coefficient, J. High Energy Phys. 02 (2015) 169.

[14] K. Landsteiner, Notes on anomaly induced transport, Acta Phys. Pol. B 47, 2617 (2016).

[15] H. Itoyama and A. H. Mueller, The axial anomaly at finite temperature, Nucl. Phys. B218, 349 (1983).

[16] F. Sannino, A note on anomaly matching for finite density QCD, Phys. Lett. B 480, 280 (2000).

[17] D. Gaiotto, A. Kapustin, N. Seiberg, and B. Willett, Generalized global symmetries, J. High Energy Phys. 02 (2015) 172.

[18] D. Gaiotto, A. Kapustin, Z. Komargodski, and N. Seiberg, Theta, time reversal, and temperature, J. High Energy Phys. 05 (2017) 091.

[19] Y. Tanizaki, Y. Kikuchi, T. Misumi, and N. Sakai, Anomaly matching for the phase diagram of massless $\mathbb{Z}_{N}$-QCD, Phys. Rev. D 97, 054012 (2018).

[20] Y. Tanizaki, Anomaly constraint on massless QCD and the role of Skyrmions in chiral symmetry breaking, J. High Energy Phys. 08 (2018) 171.
[21] Z. Wan and J. Wang, Higher anomalies, higher symmetries, and cobordisms III: QCD matter phases anew, Nucl. Phys. B957, 115016 (2020).

[22] Y. Tanizaki and M. Ünsal, Modified instanton sum in QCD and higher-groups, J. High Energy Phys. 03 (2020) 123.

[23] G. Baym, T. Hatsuda, T. Kojo, P. D. Powell, Y. Song, and T. Takatsuka, From hadrons to quarks in neutron stars: A review, Rep. Prog. Phys. 81, 056902 (2018).

[24] M. A. Metlitski and A. R. Zhitnitsky, Anomalous axion interactions and topological currents in dense matter, Phys. Rev. D 72, 045011 (2005).

[25] J. B. Kogut, M. A. Stephanov, D. Toublan, J. J. M. Verbaarschot, and A. Zhitnitsky, QCD-like theories at finite baryon density, Nucl. Phys. B582, 477 (2000).

[26] J. B. Kogut, M. A. Stephanov, and D. Toublan, On two color QCD with baryon chemical potential, Phys. Lett. B 464, 183 (1999).

[27] T. Boz, P. Giudice, S. Hands, and J.-I. Skullerud, Dense twocolor QCD towards continuum and chiral limits, Phys. Rev. D 101, 074506 (2020).

[28] K. Iida, E. Itou, and T.-G. Lee, Two-colour QCD phases and the topology at low temperature and high density, J. High Energy Phys. 01 (2020) 181.

[29] N. Astrakhantsev, V. V. Braguta, E. M. Ilgenfritz, A. Yu. Kotov, and A. A. Nikolaev, Lattice study of thermodynamic properties of dense $\mathrm{QC}_{2} \mathrm{D}$, Phys. Rev. D 102, 074507 (2020).

[30] T. Boz, O. Hajizadeh, A. Maas, and J.-I. Skullerud, Finitedensity gauge correlation functions in QC2D, Phys. Rev. D 99, 074514 (2019).

[31] T. Kojo and D. Suenaga, Thermal quarks and gluon propagators in two-color dense QCD, Phys. Rev. D 103, 094008 (2021).

[32] D. Suenaga and T. Kojo, Gluon propagator in two-color dense QCD: Massive Yang-Mills approach at one-loop, Phys. Rev. D 100, 076017 (2019).

[33] T. Kojo and G. Baym, Color screening in cold quark matter, Phys. Rev. D 89, 125008 (2014).

[34] P. V. Buividovich, D. Smith, and L. von Smekal, Numerical study of the chiral separation effect in two-color QCD at finite density, Phys. Rev. D 104, 014511 (2021).

[35] Y. Nambu, Quasiparticles and gauge invariance in the theory of superconductivity, Phys. Rev. 117, 648 (1960).

[36] D. E. Kharzeev and H. J. Warringa, Chiral magnetic conductivity, Phys. Rev. D 80, 034028 (2009).

[37] Y. Araki, D. Suenaga, K. Suzuki, and S. Yasui, Spinorbital magnetic response of relativistic fermions with band hybridization, Phys. Rev. Research 3, 023098 (2021). 
[38] D. Suenaga, Y. Araki, K. Suzuki, and S. Yasui, Chiral separation effect catalyzed by heavy impurities, Phys. Rev. D 103, 054041 (2021).

[39] G.-f. Sun, L. He, and P. Zhuang, BEC-BCS crossover in the Nambu-Jona-Lasinio model of QCD, Phys. Rev. D 75, 096004 (2007).

[40] L. He, Nambu-Jona-Lasinio model description of weakly interacting Bose condensate and BEC-BCS crossover in dense QCD-like theories, Phys. Rev. D 82, 096003 (2010).

[41] J. O. Andersen and T. Brauner, Phase diagram of two-color quark matter at nonzero baryon and isospin density, Phys. Rev. D 81, 096004 (2010).

[42] J. O. Andersen, T. Brauner, and W. Naylor, Confronting effective models for deconfinement in dense quark matter with lattice data, Phys. Rev. D 92, 114504 (2015).

[43] A. Avdoshkin, A. V. Sadofyev, and V. I. Zakharov, IR properties of chiral effects in pionic matter, Phys. Rev. D 97, 085020 (2018).

[44] J. R. Schrieffer, Theory Of Superconductivity, Advanced Books Classics (Avalon Publishing, New York, 1999).

[45] D. T. Son and Ariel R. Zhitnitsky, Quantum anomalies in dense matter, Phys. Rev. D 70, 074018 (2004).

[46] D. T. Son and M. A. Stephanov, Axial anomaly and magnetism of nuclear and quark matter, Phys. Rev. D 77, 014021 (2008).

[47] K. Jensen, Triangle anomalies, thermodynamics, and hydrodynamics, Phys. Rev. D 85, 125017 (2012).

[48] E. V. Gorbar, V. A. Miransky, I. A. Shovkovy, and X. Wang, Radiative corrections to chiral separation effect in QED, Phys. Rev. D 88, 025025 (2013).

[49] B. Feng, D.-F. Hou, and H.-C. Ren, QED radiative corrections to chiral magnetic effect, Phys. Rev. D 99, 036010 (2019).

[50] L. McLerran and R. D. Pisarski, Phases of cold, dense quarks at large N(c), Nucl. Phys. A796, 83 (2007).

[51] T. Kojo, Y. Hidaka, L. McLerran, and R. D. Pisarski, Quarkyonic chiral spirals, Nucl. Phys. A843, 37 (2010).

[52] T. Kojo, R. D. Pisarski, and A. M. Tsvelik, Covering the fermi surface with patches of quarkyonic chiral spirals, Phys. Rev. D 82, 074015 (2010).

[53] T. Kojo, A $(1+1)$ dimensional example of Quarkyonic matter, Nucl. Phys. A877, 70 (2012).

[54] T. Kojo, Y. Hidaka, K. Fukushima, L. D. McLerran, and R. D. Pisarski, Interweaving chiral spirals, Nucl. Phys. A875, 94 (2012).

[55] T. Kojo, QCD equations of state and speed of sound in neutron stars, AAPPS Bull. 31, 11 (2021).

[56] T. Kojo, P. D. Powell, Y. Song, and G. Baym, Phenomenological QCD equation of state for massive neutron stars, Phys. Rev. D 91, 045003 (2015).

[57] T. Kojo, Phenomenological neutron star equations of state: 3-window modeling of QCD matter, Eur. Phys. J. A 52, 51 (2016).

[58] G. Baym, S. Furusawa, T. Hatsuda, T. Kojo, and H. Togashi, New neutron star equation of state with quark-hadron crossover, Astrophys. J. 885, 42 (2019).

[59] T. Zhao and J. M. Lattimer, Quarkyonic matter equation of state in beta-equilibrium, Phys. Rev. D 102, 023021 (2020).

[60] K. Fukushima, T. Kojo, and W. Weise, Hard-core deconfinement and soft-surface delocalization from nuclear to quark matter, Phys. Rev. D 102, 096017 (2020).
[61] K. Sang Jeong, L. McLerran, and S. Sen, Dynamically generated momentum space shell structure of quarkyonic matter via an excluded volume model, Phys. Rev. C 101, 035201 (2020).

[62] D. C. Duarte, S. Hernandez-Ortiz, and K. Sang Jeong, Excluded-volume model for quarkyonic Matter: Three-flavor baryon-quark Mixture, Phys. Rev. C 102, 025203 (2020).

[63] D. C. Duarte, S. Hernandez-Ortiz, and K. Sang Jeong, Excluded-volume model for quarkyonic matter. II. Threeflavor shell-like distribution of baryons in phase space, Phys. Rev. C 102, 065202 (2020).

[64] Y.-L. Ma and M. Rho, Towards the hadron-quark continuity via a topology change in compact stars, Prog. Part. Nucl. Phys. 113, 103791 (2020).

[65] Y.-L. Ma and M. Rho, The sound speed and core of massive compact stars: A manifestation of hadron-quark duality, arXiv:2104.13822.

[66] I. Tews, J. Carlson, S. Gandolfi, and S. Reddy, Constraining the speed of sound inside neutron stars with chiral effective field theory interactions and observations, Astrophys. J. 860, 149 (2018).

[67] M. C. Miller et al., PSR J0030 + 0451 mass and radius from NICER data and implications for the properties of neutron star matter, Astrophys. J. Lett. 887, L24 (2019).

[68] T. E. Riley et al., A NICER view of PSR J0030 + 0451: Millisecond pulsar parameter estimation, Astrophys. J. Lett. 887, L21 (2019).

[69] NICER Collaboration, Press release (2021), https://www .nasa.gov/feature/goddard/2021/.

[70] M. C. Miller et al., The radius of PSR J0740 + 6620 from NICER and XMM-newton data, arXiv:2105.06979.

[71] T. E. Riley et al., A NICER view of the massive pulsar PSR $\mathrm{J} 0740+6620$ informed by radio timing and XMM-newton spectroscopy, arXiv:2105.06980.

[72] G. Raaijmakers, S. K. Greif, K. Hebeler, T. Hinderer, S. Nissanke, A. Schwenk, T. E. Riley, A. L. Watts, J. M. Lattimer, and W. C. G. Ho, Constraints on the dense matter equation of state and neutron star properties from NICER's mass-radius estimate of PSR J0740 + 6620 and multimessenger observations, arXiv:2105.06981.

[73] E. Fonseca et al., Refined mass and geometric measurements of the high-mass PSR J0740 + 6620, Astrophys. J. Lett. 915, L12 (2021).

[74] K. Masuda, T. Hatsuda, and T. Takatsuka, Hadron-quark crossover and massive hybrid stars with strangeness, Astrophys. J. 764, 12 (2013).

[75] K. Masuda, T. Hatsuda, and T. Takatsuka, Hadron-quark crossover and massive hybrid stars, Prog. Theor. Exp. Phys. 2013, 073D01 (2013).

[76] P. Bedaque and A. W. Steiner, Sound Velocity Bound and Neutron Stars, Phys. Rev. Lett. 114, 031103 (2015).

[77] L. McLerran and S. Reddy, Quarkyonic Matter and Neutron Stars, Phys. Rev. Lett. 122, 122701 (2019).

[78] M. Hippert, E. S. Fraga, and J. Noronha, Insights on the peak in the speed of sound of ultradense matter, Phys. Rev. D 104, 034011 (2021).

[79] T. Kojo, D. Hou, J. Okafor, and H. Togashi, Phenomenological QCD equations of state for neutron star dynamics: Nuclear-2SC continuity and evolving effective couplings, arXiv:2012.01650. 